\title{
Freqüência de aglutininas anti-Leptospira interrogans em soros sangüíneos de bovinos, em Minas Gerais, de 1980 a 2002
}

\author{
[Frequency of anti-Leptospira interrogans agglutinins in bovine serum samples \\ in Minas Gerais, Brazil, 1980 to 2002] \\ V.E.M. Araújo ${ }^{1}$, E.C. Moreira ${ }^{2}$, L.A.B. Naveda ${ }^{2}$, J.A. Silva ${ }^{2}$, R.L. Contreras ${ }^{2}$ \\ ${ }^{1}$ Secretária Municipal de Saúde - Belo Horizonte, MG \\ ${ }^{2}$ Escola de Veterinária da UFMG \\ Caixa Postal 567 \\ 30123-970 - Belo Horizonte, MG
}

\begin{abstract}
RESUMO
Foram analisados os resultados de exames de microaglutinação rápida, para pesquisa de aglutininas antiLeptospira interrogans, em 39.012 soros sangüíneos de bovinos provenientes de 398 (47\%) municípios de Minas Gerais de 1980 a 2002. As sorovariedades mais freqüentes foram: hardjo (amostra Norma), 23,7\%, hardjo (OMS), 19,7\%, hardjo (hardjobovis), 13,8\%, e wolffi, 13,2\%. Os resultados mostraram relevância da hardjo como problema prioritário nas leptospiroses em bovinos em Minas Gerais. A baixa porcentagem de soros reagentes à pomona (2,8\%) e a mini (amostra Neguita) $(3,0 \%)$ indica que essas sorovariedades devem ser consideradas para esclarecer casos clínicos em bovinos quando a hardjo (OMS), hardjo (Norma) e hardjo (hardjobovis) não forem detectadas nos testes sorológicos. As porcentagens de reagentes para as sorovariedades canicola e icterohaemorrhagiae foram inferiores a $1 \%$. A infecção por Leptospira interrogans é endêmica em bovinos em Minas Gerais.
\end{abstract}

Palavras-chave: bovino, aglutininas, Leptospira interrogans, Minas Gerais

\begin{abstract}
Blood samples from 39,012 bovines were tested for microscopic agglutination through Leptospira interrogans. An overall of 398 (47\%) of Minas Gerais State's municipalities from 1980 to 2002 composed the sample. The most frequent serovars were hardjo (Norma sample), 23.7\%, followed by hardjo (OMS sample), 19.7\%, hardjo (hardjobovis sample), 13.8\%, and wolffi, 13.2\% of positivity. The results showed the importante of hardjo serovars as a major cattle health problem in Minas Gerais. The low rates of the seropositivity for Pomona, 2.8\%, and mini (Neguita sample), 3.0\%, are an indicative which these serovars should be considered as an explanation of clinical cases in cattle when the absence of reaction for hardjo (Norma sample), hardjo (OMS sample) and hardjo (hardjobovis sample). The reaction rates for canicola and icterohaemorrhagiae serovars were below 1.0\%. The bovine infection for Leptospira interrogans is endemic in the State of Minas Gerais.
\end{abstract}

Keyword: cattle, agglutinin, Leptospira interrogans, Minas Gerais

Recebido para publicação em 9 de março de 2004

Recebido para publicação, após modificações, em 1 de março de 2005

*Autor para correspondência (corresponding author)

E-mail: elviocm@vet.ufmg.br 


\section{INTRODUÇÃO}

Vários são os problemas de sanidade que interferem na produção e na produtividade da pecuária brasileira, destacando-se o complexo da infecção por leptospiras. Essas bactérias infectam várias espécies de mamíferos silvestres e domésticos. Nos bovinos, segundo Moreira (1994), as leptospiroses causam consideráveis perdas econômicas em decorrência de repetições de cio, infertilidade, mastites, abortos, natimortalidade, bezerros prematuros, nascimento de bezerros fracos, mortes, decréscimo na produção de leite e de carne.

Sabe-se que os problemas reprodutivos atribuídos às leptospiroses em bovinos podem ocorrer sem que outra evidência clínica, como aborto, seja observada no rebanho. Portanto, o diagnóstico clínico não é conclusivo, e o veterinário precisa considerar os achados sorológicos das aglutininas antileptospiras revelados em testes de laboratório (Faine et al., 1999).

As leptospiroses fazem parte da lista B da Office International des Epizooties (OIE) de doenças a serem observadas no comércio internacional de animais e seus produtos. Segundo a OIE, ainda não se dispõe de um método de diagnóstico considerado ótimo para a determinação do status de saúde dos animais. Por essa razão, o teste de aglutinação microscópica ainda é recomendado como a melhor alternativa de diagnóstico das leptospiroses (Terrestrial, 2003).

No Brasil, as leptospiroses dos bovinos, além de não serem doenças de notificação compulsória, não estão submetidas ao combate organizado por órgãos e entidades públicas ou privadas de sanidade animal. Esse fato dificulta conhecer a verdadeira extensão das infecções por Leptospira sp. em bovinos em Minas Gerais ou em qualquer outra região do país. $\mathrm{O}$ objetivo desta investigação foi determinar a freqüência das aglutininas anti-Leptospiras interrogans nos soros sangüíneos de bovinos em Minas Gerais de 1980 a 2002.

\section{MATERIAL E MÉTODOS}

De janeiro de 1980 a dezembro de 2002, foram examinadas 39.012 amostras de soros sangüíneos de bovinos, provenientes de 398 (47\%) dos municípios de Minas Gerais. Os soros eram de fazendas com problemas reprodutivos em que a suspeita clínica era leptospirose, de centrais de inseminação artificial, matadouros, plantéis que produzem bovinos destinados à exportação e de propriedades com assistência veterinária em que a sorologia para as leptospiroses bovinas era feita, em conjunto com outros testes, para monitoramento de problemas sanitários.

Nos testes sorológicos, foram utilizadas, como antígenos, culturas vivas de Leptospira interrogans, cedidas pelo antigo Centro Panamericano de Zoonoses da Organização Panamericana da Saúde-Argentina. As aglutininas antileptospiras foram pesquisadas pelo método de microaglutinação rápida (MAR) descrito por Ryu (1970), e o critério adotado para leitura das reações positivas nos soros foi a diluição de $1: 100$, cujo campo microscópico apresentou $50 \%$ ou mais de leptospiras aglutinadas.

O número de sorovariedades utilizadas, na bateria de antígenos, variou ao longo da série histórica. A sorovariedade brasiliensis foi incluída em 1991 e excluída da bateria de antígenos em 1994. As sorovariedades hardjo (Norma), szwajizak e mini (Neguita) foram incluídas nos testes sorológicos a partir de 1997. A hardjo (hardjobovis) foi incluída a partir de 1998 (Tab. 1).

A base de dados foi construída com o auxílio do programa Epi Info versão $6.04 \mathrm{~b}$; para cada amostra, foram registradas as datas da coleta, as sorovariedades utilizadas na bateria de antígenos e os resultados das reações de microaglutinação observadas. As fichas que não continham todas essas informações foram excluídas.

Os resultados das pesquisas de aglutininas antileptospiras para cada sorovariedade foram submetidos à análise descritiva, com auxílio do Epi Info versão $6.04 \mathrm{~b}$, definindo-se as freqüências absolutas e relativas de bovinos testados e reagentes em Minas Gerais, no período de 1980 a 2002.

Para avaliar as diferenças entre as freqüências observadas para as sorovariedades hardjo (genótipo Hardjoprajitno - amostra Norma), hardjo (genótipo Hardjoprajitno - amostra 
OMS), hardjo (hardjobovis) e wolffi, utilizou-se o teste do qui-quadrado (Sampaio, 2002) com nível de significância $\mathrm{P}<0,05$.

\section{RESULTADOS E DISCUSSÃO}

A freqüência de aglutininas antileptospiras, em soros sangüíneos de bovinos em Minas Gerais, de acordo com as sorovariedades, de 1980 a 2002, é apresentada na Tab. 2.

As sorovariedades mais freqüentes nos testes de MAR foram hardjo (Norma), hardjo (OMS), hardjo (hardjobovis) e wolffi. As sorovariedades hardjo (Norma), szwajizak e mini (Neguita) foram incluídas nos testes sorológicos em 1997 e a hardjo (hardjobovis), em 1998. Isso explica o menor número de soros testados para essas sorovariedades em comparação com o grande número de soros testados para hardjo (OMS), wolffi, pomona e grippothyphosa que constam da coleção de antígenos desde 1980. As porcentagens de soros sangüíneos reagentes para ballum, sejroe, autumnalis, butembo, canicola, icterohaemorrhagiae, grippothyphosa e australis foram inferiores a $1 \%$.

As sorovariedades hardjo (Norma), hardjo (OMS) e hardjo (hardjobovis) são reconhecidas como patogênicas para os bovinos, principalmente como causas de transtornos reprodutivos. O papel da wolffi na infecção em bovinos, desde a exaustiva revisão feita por Amatredjo e Campbell (1975) até a publicação de Faine et al. (1999), não está bem definido. Até o momento, não foram encontradas publicações científicas e/ou técnicas com registros do isolamento de wolffi em bovinos nos casos clínicos sugestivos de leptospiroses. Outra característica que deve ser considerada para interpretar o papel da wolffi em bovinos no Brasil é sua afinidade antigênica com as sorovariedades hardjo (Norma, OMS e hardjobovis), revelada pela alta taxa de reações cruzadas nos testes de microaglutinação, como assinalam Costa et al. (1998).

Tabela 1. Sorovariedades de Leptospira interrogans utilizadas como antígenos no teste de microaglutinação rápida

\begin{tabular}{|c|c|c|}
\hline Sorogrupo & Sorovariedade & Amostra de referência \\
\hline \multirow[t]{2}{*}{ Australis } & australis & Ballico \\
\hline & bratislava & Jez Bratislava \\
\hline Autumnalis & autumnalis & Akiyami A \\
\hline Ballum & ballum & Mus 127 \\
\hline \multirow[t]{2}{*}{ Bataviae } & bataviae & Swart \\
\hline & brasiliensis $^{\text {a }}$ & An 776 \\
\hline Butembo & butembo & Butembo \\
\hline Canicola & canicola & Hond Utrech IV \\
\hline Grippothyphosa & grippothyphosa & Moskva V \\
\hline Hebdomadis & hebdomadis & Hebdomadis \\
\hline Icterohaemorrhagiae & icterohaemorrhagiae & RGA \\
\hline \multirow[t]{2}{*}{ Mini } & szwajizak $^{\text {b }}$ & Szwajizak \\
\hline & $\operatorname{mini}^{b}$ & Neguita \\
\hline Pomona & pomona & Pomona \\
\hline Pyrogenes & pyrogenes & Salinem \\
\hline \multirow[t]{5}{*}{ Sejroe } & hardjo & Hardjoprajitno (OMS) \\
\hline & hardjo $^{b}$ & Hardjoprajitno (Norma) \\
\hline & hardjo (hardjobovis) $^{c}$ & Sponselee \\
\hline & wolffi & 3705 \\
\hline & sejroe & M 84 \\
\hline Tarassovi & tarassovi & Perepelicin \\
\hline
\end{tabular}

a incluída em 1991 e excluída em 1994; ${ }^{b}$ incluídas em 1997; ${ }^{\text {c }}$ incluída em 1998. 
Freqüência de aglutininas anti-Leptospira interrogans...

Tabela 2. Aglutininas anti-Leptospira interrogans em soros sangüíneos de bovinos, Minas Gerais, Brasil, 1980 a 2002

\begin{tabular}{|c|c|c|c|}
\hline \multirow{2}{*}{ Sorovariedade } & \multicolumn{2}{|c|}{ Número de soros } & \multirow{2}{*}{ Porcentagem } \\
\hline & Testados & Reagentes & \\
\hline hardjo (Norma) & 8317 & 1970 & 23,7 \\
\hline hardjo (OMS) & 38883 & 7653 & 19,7 \\
\hline hardjobovis & 2698 & 372 & 13,8 \\
\hline wolffi & 38710 & 5098 & 13,2 \\
\hline bratislava & 24288 & 1428 & 5,9 \\
\hline bataviae & 24278 & 1174 & 4,9 \\
\hline szwajizak & 2980 & 141 & 4,7 \\
\hline tarassovi & 26342 & 871 & 3,3 \\
\hline $\operatorname{mini}$ & 5648 & 166 & 2,9 \\
\hline pomona & 38581 & 1069 & 2,8 \\
\hline pyrogenes & 23251 & 465 & 2,00 \\
\hline hebdomadis & 25422 & 444 & 1,7 \\
\hline ballum & 17058 & 130 & 0,8 \\
\hline sejroe & 786 & 5 & 0,6 \\
\hline autumnalis & 21148 & 113 & 0,5 \\
\hline butembo & 16923 & 82 & 0,5 \\
\hline canicola & 25300 & 115 & 0,5 \\
\hline icterohaemorrhagiae & 18835 & 79 & 0,4 \\
\hline grippothyphosa & 30842 & 58 & 0,2 \\
\hline australis & 18695 & 21 & 0,1 \\
\hline brasiliensis & 310 & 0 & 0,0 \\
\hline
\end{tabular}

$\chi^{2}=73,26(\mathrm{P}<0,001)$ : valor referente às sorovariedades hardjo (Norma) e hardjo (OMS).

$\chi^{2}=881,9(\mathrm{P}<0,001)$ : valor referente às sorovariedades hardjo (Norma), hardjo (OMS), hardjo (hardjobovis) e wolffi.

A brasiliensis foi excluída da coleção de antígenos por não apresentar soros reagentes entre os 310 testados. Provavelmente, essa sorovariedade não tem relevância para a bovinocultura nacional, pois o inquérito sorológico realizado em Roraima (Zelada, 1981) não detectou soro reagente. Moreira (1982), no Pará, observou somente $0,3 \%$ de aglutininas para essa sorovariedade. Em São Luís, Santos (1988) verificou a ausência de soros reagentes para brasiliensis e o predomínio de aglutininas antiballum (38,3\%), seguida de hardjo $(19,1 \%)$ e wolffi $(16,7 \%)$. A elevada taxa de reagentes para ballum foi atribuída à criação dos bovinos em ambiente urbano.

O cálculo de $\chi^{2}$ considerando os resultados observados e esperados para hardjo (Norma) $(23,7 \%)$, hardjo (OMS) (19,7\%), hardjo (hardjobovis) $(13,8 \%)$ e wolffi $(13,2 \%)$ revelou alto nível de significância $(\mathrm{P}<0,001)$. Tal resultado sugere que essas diferentes porcentagens de reagentes não são aleatórias e estariam associadas às propriedades antigênicas de cada uma dessas quatro sorovariedades. Assim, pode-se afirmar $(\mathrm{P}<0,001)$ que a sorovariedade hardjo (genótipo Hardjoprajitno - amostra Norma) é a mais freqüente no grupo de bovinos testados. Esses valores representam a provável distribuição das sorovariedades de leptospiras que infectam os bovinos em Minas Gerais.

Os inquéritos sorológicos feitos em Minas Gerais (Moreira et al., 1979; Ribeiro et al., 1988 a, b; Ribeiro et al., 2000), no Mato Grosso (Madruga et al., 1980), no Rio de Janeiro (Lilenbaum et al., 1995), em São Paulo (Vasconcelos et al., 1997; Langoni et al., 2000 ), em Pernambuco ( Oliveira et al., 2001) e em áreas tropicais (Ellis, 1984) também encontraram elevada freqüência de bovinos reagentes para hardjo. $\mathrm{O}$ isolamento de hardjo (genótipo Hardjoprajitno - amostra Norma) em bovinos de Minas Gerais por Moreira (1994) confirma a relevância dessa sorovariedade como problema prioritário das leptospiroses em bovinos no Brasil.

A alternativa mais eficaz para controlar ou erradicar as leptospiroses em bovinos é o uso de vacina elaborada com a sorovariedade específica prevalente na propriedade. No Brasil, as nove vacinas disponíveis no mercado não atendem a esse pré-requisito. Como divulgado no Manual 
de Produtos Veterinários (Manual..., 2002), elas contêm, em sua composição, as sorovariedades canicola, icterohaemorrhagiae, tarassovi, grippothyphosa, wolffi e bratislava. Os resultados desta pesquisa revelaram ausência dessas sorovariedades nos bovinos criados em Minas Gerais.

Porcentagens iguais ou inferiores a $1 \%$, de qualquer sorovariedade que infecta bovinos, podem ser consideradas como reações cruzadas quando não existe registro de isolamento. Assim, os bovinos que foram reagentes para canicola e icterohaemorrhaghiae apresentaram freqüências baixas e próximas dos achados de outros pesquisadores, como Ribeiro et al. (1988 a) e Ribeiro et al. (2000), em Minas Gerais, e Oliveira et al. (2001), em Pernambuco.

\section{CONCLUSÕES}

A infecção por Leptospira sp. é endêmica em bovinos em Minas Gerais, demonstrada pela presença de aglutininas antileptospiras nos soros sangüíneos. A sorovariedade hardjo (amostra Norma) é a principal leptospira que infecta bovinos em Minas Gerais, seguida de hardjo (amostra OMS) e hardjo (hardjobovis). As sorovariedades pomona e mini (amostra Neguita) com baixa porcentagem de bovinos reagentes ainda devem fazer parte da bateria de antígenos para esclarecer casos clínicos em bovinos compatíveis com lepstospiroses e que foram negativos para sorovariedades hardjo (Norma, OMS e bovis).

\section{REFERÊNCIAS BIBLIOGRÁFICAS}

AMATREDJO, A.; CAMPBELL, R.S.F. Bovine leptospiroses. Vet. Bull., v.43, p.875-891, 1975.

COSTA, M.C.R.; MOREIRA, E.C.; LEITE, R.C. et al. Avaliação da imunidade cruzada entre Leptospira hardjo e L. wolffi. Arq. Bras. Med. Vet. Zootec., v.50, p.11-17, 1998.

ELLIS, W.A. Bovine leptospiroses in the tropics: prevalence, pathogenesis and control. Prev. Vet. Med., v.2, p.411-421, 1984.

EPI Info, version 6: a word processing, database and statistics program for public health on IBM compatible microcomputers. Atlanta: Centers for Disease Control and Prevention, 1995. 686p.
FAINE, S.; ADLER, B.; BOLIN, C. et al. Leptospira and leptospirosis. 2.ed. Melbourne: MediSci, 1999. 272p.

LANGONI, H.; MEIRELES, L.R.; GOTTSCHALK, S. et al. Perfil sorológico da leptospirose bovina em regiões do Estado de São Paulo. Arq. Inst. Biol. São Paulo, v.67, p.37-41, 2000.

LILENBAUM, W.; SANTOS, M.R.C.; BARBOSA, A.V. Leptospirose em reprodução animal: II. Bovinos do estado do Rio de Janeiro, Brasil. Rev. Bras. Ciênc. Vet., v.2, p.1-6, 1995.

MADRUGA, C.R.; AYCARDI, E.; PUTT, N. Freqüência de aglutininas anti-leptospira em bovinos de corte da região sul de cerrado do estado de Mato Grosso. Arq. Esc. Vet. UFMG., v.32, p.245-249, 1980.

MANUAL de produtos veterinários. Brasília: Ministério da Agricultura, Pecuária e Abastecimento. 2002. 1106p.

MOREIRA, E.C. Avaliação de métodos para erradicação de leptospiroses em bovinos leiteiros. 1994. 93f. Tese (Doutorado)- Escola de Veterinária, Universidade Federal de Minas Gerais, Belo Horizonte.

MOREIRA, E.C.; SILVA, J.A.; VIANA, F.C. et al. Leptospirose bovina: I - aglutininas anti-leptospiras em soros sangüíneos de bovinos em Minas Gerais. Arq. Esc. Vet. UFMG., v.31, p.375-388, 1979.

MOREIRA, T.M.S. Prevalência de aglutininas antileptospiras em soros sangüíneos de bovinos dos Estados do Pará e Amazonas. 1982. 43f. Dissertação (Mestrado) - Escola de Veterinária, Universidade Federal de Minas Gerais, Belo Horizonte.

OLIVEIRA, A.A.F.; MOTA, R.A.; PEREIRA, G.C. et al. Seroprevalence of bovine leptospirosis em Garanhuns municipal district, Pernambuco State, Brazil. Onderstepoort J. Vet. Res., v.68, p.275-279, 2001.

RIBEIRO, S.C.A.; BISINOTO, D.P.; OLIVEIRA, P.R. Prevalência da leptopspirose em fêmeas reprodutoras bovinas do município de Uberlândia, MG. Vet. Not., v.6, p.69-75, 2000.

RIBEIRO, S.C.A.; GOUVEIA, M.A.V.; SILVA, P.L. et al. Levantamento sorológico em dois surtos de leptospirose bovina em Uberlândia, Triângulo Mineiro. Arq. Bras. Med. Vet. Zootec., v.40, p.415423, 1988a.

RIBEIRO, S.C.A.; MOREIRA, E.C.; GOMES, A.G. et al. Infecção por Leptospira interrogans, numa fazenda de Minas Gerais, Brasil. Arq. Bras. Med. Vet. Zootec., v.40, p.137-144, 1988b.

RYU, E. Rapid microscopic agglutination test for Leptospira based on 400X magnification of darkfield 
Freqüência de aglutininas anti-Leptospira interrogans...

examination. Taiwan J. Vet. Med. Anim. Husb., n.17, p.1-9, 1970.

SAMPAIO, I.B.M. Estatística aplicada à experimentação animal. 2.ed. Belo Horizonte: Fundação de Estudo e Pesquisa em Medicina Veterinária e Zootecnia, 2002. 133p.

SANTOS, H.P. Alguns aspectos do sistema de produção e da sanidade dos bovinos de leite da Ilha de São Luiz - MA. 1988. 91f. Dissertação (Mestrado) - Escola de Veterinária, Universidade Federal de Minas Gerais, Belo Horizonte.

TERRESTRIAL animal health code. Disponível em: $<$ htttp://www.oie.int/eng/normes/en_mcode.htm>. Acessado em: 22/07/2003.
VASCONCELLOS, S.A.; BARBARINI JÚNIOR, O.; UMEHARA, O. et al. Leptospirose bovina. Níveis de ocorrência e sorotipos predominantes em rebanhos dos estados de Minas Gerais, São Paulo, Rio de Janeiro, Paraná, Rio Grande do Sul e Mato Grosso do Sul. Arq. Inst. Biol. São Paulo, v.64, p.7-15, 1997.

ZELADA, H.M. Prevalência de aglutininas antileptospiras em soros sangüineos de bovinos do território federal de Roraima - Brasil. 1981. $43 \mathrm{f}$. Dissertação (Mestrado) - Escola de Veterinária, Universidade Federal de Minas Gerais, Belo Horizonte. 